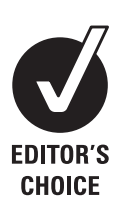
p 856

${ }^{1}$ Department of Safety of Medicinal Products and Medical Devices, Paul-Ehrlich-Institute, Federal Institute for Vaccines and Biomedicines, Langen, Germany

${ }^{2}$ Institute of Virology, Heinrich-Heine-University Duesseldorf, Duesseldorf, Germany

${ }^{3}$ Department of Neurology, St. Josef Hospital, Ruhr University Bochum, Bochum, Germany ${ }^{4}$ Department of Neurology, Heinrich-Heine-University Duesseldorf, Duesseldorf, Germany

${ }^{5}$ Department of Haematology, Oncology and Tumour Immunology, HELIOS Clinic Berlin-Buch, Berlin, Germany

Correspondence to Dr Dirk Mentzer, Paul-Ehrlich-Institute, Federal Institute for Vaccines and Biomedicines, Department of Safety of Medicinal Products and Medical Devices, Paul-Ehrlich-Str. 51-59, 63225 Langen, Germany; dirk.mentzer@pei.de

Received 9 February 2012 Revised 29 April 2012 Accepted 16 May 2012 Published Online First 17 July 2012

\title{
Case definition for progressive multifocal leukoencephalopathy following treatment with monoclonal antibodies
}

\author{
Dirk Mentzer, ${ }^{1}$ Jürgen Prestel, ${ }^{1}$ Ortwin Adams, ${ }^{2}$ Ralf Gold ${ }^{3}$ Hans-Peter Hartung, ${ }^{4}$ \\ Hartmut Hengel, ${ }^{2}$ Bernd C Kieseier, ${ }^{4}$ Wolf-Dieter Ludwig, ${ }^{5}$ \\ Brigitte Keller-Stanislawski ${ }^{1}$
}

\section{ABSTRACT}

Background Novel immunosuppressive/modulating therapies with monoclonal antibodies (MABs) have been associated with progressive multifocal

leukoencephalopathy (PML), a potentially fatal disease of the brain caused by the JC virus. Taking the complex diagnostic testing and heterogeneous clinical presentation of PML into account, an agreed case definition for PML is a prerequisite for a thorough assessment of PML.

Objective/methods A working group was established to develop a standardised case definition for PML which permits data comparability across clinical trials,

postauthorisation safety studies and passive postmarketing surveillance. The case definition is designed to define levels of diagnostic certainty of reported PML cases following treatment with MABs. It was

subsequently used to categorise retrospectively suspected PML cases from Germany reported to the Paul-EhrlichInstitute as the responsible national competent authority. Results The algorithm of the case definition is based on clinical symptoms, PCR for JC virus DNA in cerebrospinal fluid, brain MRI, and brain biopsy/autopsy. The case definition was applied to 119 suspected cases of PML following treatment with MABs and is considered to be helpful for case ascertainment of suspected PML cases for various MABs covering a broad spectrum of indications. Even if the available information is not yet complete, the case definition provides a level of diagnostic certainty. Conclusions The proposed case definition permits data comparability among different medicinal products and among active as well as passive surveillance settings. It may form a basis for meaningful risk analysis and communication for regulators and healthcare professionals.

\section{INTRODUCTION}

Progressive multifocal leukoencephalopathy (PML) is a rare, potentially fatal, demyelinating disease of the human brain caused by the JC virus (JCV), a human polyomavirus. It is thought that PML occurs following reactivation of JCV in the setting of severe cellular immune deficiency. Reactivation of JCV causes a lytic infection of oligodendrocytes and destruction of brain tissue.

Most investigators have found that JCV infection often occurs during childhood, apparently without clinical symptoms. Seroprevalence rates vary in the literature based on the population and ethnical origin investigated. Egli et a ${ }^{1}$ reported a seroprevalence rate of $58 \%$ in healthy adults consistent with recent prevalence data of anti-JCV antibodies determined in multiple sclerosis (MS) patients. ${ }^{2}$

PML is a well-known complication in immunosuppressed patients, particularly in those with AIDS, haematological malignancies (eg, lymphoproliferative disorders), organ or haematopoietic stem cell transplantations, and those exposed to antineoplastic or immunosuppressive therapies. While the aspects that trigger JCV pathogenicity are not known, it has been assumed that viral mutations increase pathogenicity. ${ }^{3}$

Novel immunosuppressive/modulating therapies with monoclonal antibodies (MABs), for example, natalizumab, rituximab, alemtuzumab, efalizumab and brentuximab vedotin, have been associated with PML. ${ }^{4-6}$

The early neurological symptoms may be nonspecific or subtle and dependent on the initial focus of the reactivation in the brain. Thus, it may be difficult to differentiate first symptoms of PML from the underlying disease for which $M A B s$ are used (particularly relapse/progression of MS or-as a much less frequent use-central nervous system (CNS) systemic lupus erythematosus ${ }^{7}$ ). In addition, first clinical symptoms of PML in patients treated with $\mathrm{MABs}$ for lymphoma may initially be suspected as CNS involvement by lymphoma. ${ }^{8}$ Besides, concomitant diseases with neurological symptoms such as other neuroinflammatory conditions, stroke or cerebral malignancies may mimic clinical signs suspicious of PML.

Considering a high level of awareness of the PML risk among physicians, many cases may be reported as suspected PML cases at the onset of new neurological symptoms. Thus, physicians may need to monitor a patient over weeks with repeated MRI and cerebrospinal fluid (CSF) testing, as well as continuing to watch for clinical progression or even performing brain biopsy, before the diagnosis of PML can be finally confirmed or rejected. As a consequence, initial reports by physicians may not contain all the necessary information for final ascertainment. Furthermore, passive surveillance reporting may not be as complete as reporting from studies. However, for signal detection and risk analysis it is not acceptable to disregard or underestimate less complete reports. 


\section{Objective}

Taking the complex diagnostic testing (eg, MRI, PCR) and heterogeneous clinical presentation of PML into account, an agreed case definition for PML is a prerequisite for a thorough assessment of PML. This is also acknowledged by European Union and US regulators. ${ }^{9}$ The aim was to develop a standardised case definition for PML associated with MABs in order to enhance data comparability across different clinical trials, passive surveillance systems and postauthorisation safety studies. The case definition is designed to define the levels of diagnostic certainty of reported PML cases following treatment with MABs.

\section{METHODS}

A working group of experts in neurology, virology, haematology and pharmacovigilance was established to assess and discuss their experience with the diagnosis and treatment of PML. A case definition was developed based on the exploratory review of literature and group consensus. Analogous to the model of the Brighton Collaboration case definition, ${ }^{10}$ level 1 reflects the highest level of diagnostic certainty, level 2 reflects an intermediate and level 3 a low level of diagnostic certainty but a high level of sensitivity. Level 4 means that the evidence available for a reported event of PML is insufficient to permit classification at any level of diagnostic certainty (eg, information to confirm with certainty or to exclude PML is still missing). Level 5 reflects the exclusion of the initial PML suspicion. For pharmacovigilance purposes, a case is considered as PML if the description meets the levels $1-3$ criteria. Although the case definition is independent from the causality assessment between PML and the medicinal product, it is considered to be a prerequisite for causality assessment.

In a second step, the proposed case definition was retrospectively applied to all suspected German PML cases reported to the Paul-Ehrlich-Institute (PEI). Reporting of suspected PML cases to the PEI as the responsible national competent authority is mandatory for marketing authorisation holders according to the German Medicinal Products Act and European Regulation 726/ 2004 EC. Elements for reporting are described in the guideline E2B(R2) of the International Conference on Harmonisation. ${ }^{11}$ In Germany, physicians and pharmacists are obliged to report suspected adverse reactions to the respective professional commissions (Drug Commission of the German Medical Association/ of German Pharmacists) which exchange information with the PEI. In order to retrieve reported suspected cases of PML, the PEI adverse drug reaction database was queried against the MedDRA (medical dictionary for regulatory activities) preferred terms 'progressive multifocal leukoencephalopathy' (10036807), 'JC virus infection' (10023163), 'human polyomavirus infection' (10057366), 'leukoencephalopathy' (10024382) and 'leukoencephalomyelitis' (10048999). The result of this query was manually evaluated thereafter. Each chart was reviewed and the case definition was retrospectively applied to each reported suspected PML case. Patient characteristics and first symptoms of PML were described.

\section{CASE DEFINITION}

The following diagnostic key elements have been translated into the case definition by the working group.

\section{Clinical symptoms}

The presence of new focal neurological deficits that may be subacute during onset may point towards PML. Cognitive dysfunction (eg, confusion) or recent changes in behaviour or personality, motor symptoms (eg, hemiparesis or focal paresis), language or speech disturbances (aphasia/dysarthria), visual disturbance (eg, hemianopsia), ataxia/loss of motor coordination and seizures have been frequently reported as first symptoms of natalizumab-associated PML. ${ }^{12}{ }^{13}$ Common presenting symptoms of PML in patients treated with rituximab included cognitive dysfunction (confusion, disorientation), motor weakness/hemiparesis, poor motor coordination/ataxia, language/ speech difficulties or visual symptoms. ${ }^{5}{ }^{14}$ Similarly, in efalizumab-associated PML, patients often displayed memory difficulties/altered mental status, changes in behaviour or mood, motor weakness, or language/speech difficulties. ${ }^{15} 16$ However, less frequently, other symptoms have also been reported as presenting clinical features (eg, sensory symptoms in patients treated with natalizumab, ${ }^{13}$ rituximab $^{14}$ or efalizumab, ${ }^{15}$ or myoclonus in a natalizumab- ${ }^{12}$ or rituximab-treated patient ${ }^{14}$ ). As clinical symptoms of PML are not pathognomonic, differential diagnosis for new neurological symptoms needs to be thoroughly performed (eg, MS relapse or other neuroinflammatory conditions (such as neuropsychiatric lupus erythematosus or CNS vasculitis), other neuroinfectious conditions (such as HIV encephalitis/HIV encephalopathy or opportunistic infections, eg, toxoplasmosis), stroke, brain tumour, CNS lymphoma).

In particular, it may be challenging to differentiate symptoms of PML from those of an acute MS relapse. However, in contrast to relapsing-remitting MS, PML shows slowly progressive focal neurological deficits.

\section{MRI}

A main pillar for the diagnostic confirmation beside the progressive clinical deterioration is the MRI. A standard cranial MRI protocol such as proposed for natalizumab ${ }^{17}$ is important for diagnostic sensitivity and specificity. Some particularly important aspects are (1) scanner field strength $\geq 1.0 \mathrm{~T}$, (2) slice thickness $\leq 5 \mathrm{~mm}$ covering the whole brain without interslice gap, (3) scan orientation on the subcallosal line and exact repositioning (three-plane localiser), (4) fluid attenuated inversion recovery (FLAIR) sequences (axial and sagittal, if possible) and proton density-weighted sequences apart from standard T2weighted sequences and (5) administration of contrast medium (gadolinium) in a standardised protocol of T1-weighted imaging with more than 5 min delay (and at most 10 min delay) after contrast injection.

As seen on MRI, single or multifocal PML lesion(s) frequently affect the subcortical hemispheric white matter with involvement of $U$ fibres, but the lesions might also be located, for example, periventricular or in the white matter of the cerebellar hemispheres or peduncles. Although PML lesions typically involve the white matter, grey matter involvement can occur. PML lesions are often asymmetric, particularly in the early stage of the disease. Normally, there is no mass effect in PML lesions in contrast to some acute tumefactive MS lesions. PML lesions are (partially) hypointense on T1-weighted MR imaging and hyperintense on T2-weighted images, FLAIR images and proton density-weighted images. Newer lesions and the advancing edge of large lesions have a high signal on diffusion-weighted images, with reduced signal intensity typically being seen in the core of these lesions. $^{7} 12141819$

Unlike in classic PML, where gadolinium enhancement in MRI is usually minimal or absent, 12 of 28 natalizumab-treated patients, who developed PML, had gadolinium enhancement at the time of diagnosis. ${ }^{13}$ However, there is no evidence that this 


\section{Box 1 Levels 1-5 of diagnostic certainty of PML case definition}

\section{Level 1 of diagnostic certainty}

- Evidence of PML from a brain biopsy or autopsy*

\section{OR}

- Clinical symptoms consistent with PML†

AND

- Brain MRI characteristic of PML‡

AND

- PCR for JCV DNA in CSF positive§

\section{Level 2 of diagnostic certainty}

- Clinical symptoms consistent with PML $†$

AND

- PCR for JCV DNA in CSF positive§

\section{Level 3 of diagnostic certainty}

- Clinical symptoms consistent with PML†

AND

- Brain MRI characteristic of PML

AND

- PCR for JCV DNA in CSF or brain biopsy/autopsy not available OR PCR assay for JCV DNA in CSF obtained by a laboratory with unknown virological expertise or unknown validation status of assay

\section{OR}

- PCR for JCV DNA in CSF positive§

AND

- Brain MRI characteristic of PML $\ddagger$

AND

- Clinical symptoms unclear, not reported, or no clinical symptoms

\section{OR}

$\checkmark$ PCR for JCV DNA in CSF positive§

AND

- IRIS (eg, after stopping treatment with suspected medicinal product and/or after therapy)

AND

- Clinical symptoms unclear or not reported, MRI not available or non-specific (may also be consistent with MS relapse)

Reported event of PML with insufficient evidence to permit classification at any level of diagnostic certainty (level 4)

- Cases not fulfilling levels 1-3 of diagnostic certainty (eg, because of missing information) AND not meeting the exclusion criteria (level 5)

\section{PML excluded (level 5)}

PML can be excluded only if the following criteria are considered to be fulfilled:

- Neurological clinical assessment: Alternative diagnosis established by treating neurologist based on the clinical symptoms and course AND at least one of the following diagnostic criteria:

- Absence of characteristic features of PML on brain MRI

(MRI not characteristic for PML as described in a radiological report $O R$ based on expert review of MRI)

- PCR for JCV DNA in CSF negative§

- Brain biopsy or autopsy negative for PML

*Evidence of PML from a brain biopsy or autopsy: Histopathological evidence (demyelination, enlarged oligodendroglial cells, bizarre astrocytes) in addition to immunohistochemical (JCV large T Ag and JCV VP1 capsid protein) or electron microscopic (JCV virions) evidence.

†Clinical symptoms of PML: Presence of focal neurological deficits, including new deficits, that may be subacute in onset, or worsening of deficits; clinical symptoms may include at least one of the following symptoms: cognitive dysfunction, recent changes in behaviour or personality or mood, hemiparesis (or focal paresis), language disturbance (aphasia) or speech difficulties (dysarthria), retrochiasmal visual deficits, ataxia, and/or new onset of seizures.

¥Characteristic PML findings on MRI as described in a radiological report $O R$ based on expert review of MRI.

§PCR for JCV DNA in CSF should always be obtained by a laboratory with specific virological expertise and a validated assay.

CSF, cerebrospinal fluid; IRIS, immune reconstitution inflammatory syndrome; JCV, JC virus; PML, progressive multifocal leukoencephalopathy. 
finding in natalizumab-associated PML can be generalised to PML associated with other MABs. For natalizumab-treated patients, this finding implies that-based on the fact of a contrast enhancement alone-a differentiation between PML lesion and MS lesion may not be possible. However, the pattern of contrast enhancement in MS lesions is often homogeneous with sharp edges or ring-like, whereas PML lesions can show an inhomogeneous, cloudy, speckled, ribbon-like or ring-like enhancement. In immune reconstitution inflammatory syndrome (IRIS), PML lesions might show (increased) contrast enhancement which might also afflict previously normalappearing white matter. Contrast enhancement in patients with IRIS might be detected in natalizumab-associated $\mathrm{PML}^{20}$ rituximab-associated $\mathrm{PML}^{14}$ and in AIDS-associated PML. ${ }^{21}$

As a consequence, differentiation between PML lesion and MS lesion with certainty based on MRI alone is often not possible, particularly not in the early stage of disease. Thus, the diagnosis of PML cannot be made with certainty based on clinical symptoms and MRI findings alone, because both are not pathognomonic, and the differential diagnosis may still include MS exacerbation (in patients treated with natalizumab) or other conditions (eg, stroke), particularly in the early stage of disease.

\section{Virological and histopathological testing}

The confirmation of a suspected case of PML requires the presence of JCV DNA in the CSF by PCR or a histopathological confirmation in a brain biopsy/autopsy.

When the suspicion of PML persists after physical examination and MRI, a PCR for JCV DNA in the CSF is obligatory. The PCR should be conducted in a laboratory with specific virological expertise. A real-time PCR should be performed to ensure maximal sensitivity and specificity for detection. However, a negative JCV PCR result does not exclude PML (CSF can be negative for JCV DNA despite clinical and MRI findings, eg, in early PML or during IRIS). ${ }^{22}$ If the suspicion of PML persists, PCR for JCV DNA in the CSF should be repeated. ${ }^{23}$ Testing for JCV DNA in serum, plasma or urine is not useful for making or excluding a PML diagnosis and has no diagnostic value regarding PML. $^{2}$

However, the presence of only JCV DNA in the CSF without clinical symptoms and without MRI features consistent with the diagnosis may be insufficient to confirm a case of PML. For example, in a PCR analysis ${ }^{24}$ of 210 cell-free CSF samples and of 42 CSF cell samples, each from MS patients, a low copy number of JCV DNA was detected in one cell-free CSF sample (1/210, $0.5 \%$ ) and in one CSF cell sample (1/42, 2.4\%). These two patients have been followed up clinically for 24 and 30 months, respectively, without developing any signs of PML. These data show that even with a validated PCR method in an experienced laboratory with specific virological expertise, a low copy number of JCV DNA can be detected in the CSF of persons without PML in rare cases. Thus, a diagnosis of PML must rely on a combined assessment of clinical picture, brain MRI and JCV PCR in CSF. For cases with incomplete or insufficient information, follow-up data need to be diligently sought to allow a classification of the case.

In rare instances (eg, repeatedly negative JCV DNA PCR in CSF and clinically persisting suspicion of PML), a brain biopsy needs to be taken into consideration. Histological PML features include focal areas of demyelination, enlarged oligodendrocytes and large 'bizarre-looking' astrocytes.

By immunohistochemistry, JCV proteins (large T antigen and VP1 capsid protein) are detectable on a brain biopsy sample of a PML lesion in most cases. In a recent study, ${ }^{25} \mathrm{JCV}$ large $\mathrm{T}$ antigen was found in brain samples of 21/24 (88\%) HIV-positive PML patients, VP1 capsid protein in brain samples of $20 / 24$ (83\%) HIV-positive PML patients and in 23/24 (96\%) HIVpositive PML brain samples either one or both of these JCV proteins were expressed. In the same study, large $T$ antigen (but not VP1 capsid protein) was found in one brain sample each from a HIV-positive (1/18, 6\%) and HIV-negative (1/19, 5\%) control group without PML.

A positive histological examination and an immunohistochemical detection of JCV proteins are required to provide evidence for PML from a brain biopsy (or alternatively a positive histological and electron microscopic examination). JCV DNA can be detected by in situ hybridisation in the brain of individuals without PML. ${ }^{25}$ Thus, the sole detection of JCV DNA in a brain biopsy is not sufficient to provide evidence for a PML.

Key elements of the case definition are presented in box 1 . The algorithm is based on clinical symptoms, PCR testing, MRI and brain biopsy/autopsy. Although the clinical presentation is not pathognomonic, clinical symptoms usually trigger a subsequent diagnostic work-up and are therefore considered as a key element for the case definition. It is acknowledged that the pathological evaluation (brain biopsy/examination of brain tissue) is the most specific diagnostic tool, but its use is limited.

\section{APPLICATION OF PML CASE DEFINITION}

Up to 27 July 2011, a total of 119 suspected cases of PML in association with MABs in Germany were reported to the PEI. Of those, 94 suspected cases were reported as postmarketing spontaneous reports, 22 from postmarketing studies and three

Table 1 Assessment according to the proposed case definition $(n=119)$

\begin{tabular}{|c|c|c|c|c|c|c|c|}
\hline MAB & Underlying disease (as reported) & Total number & Level 1 & Level 2 & Level 3 & Level 4 & Level 5 \\
\hline Natalizumab & MS & $72(100 \%)$ & $25(34.7 \%)$ & $1(1.4 \%)$ & $1(1.4 \%)$ & $16(22.2 \%)$ & $29(40.3 \%)$ \\
\hline \multirow[t]{5}{*}{ Rituximab } & Haematological malignancies & 32 & 4 & 1 & 9 & 18 & - \\
\hline & RA & 2 & - & - & - & 2 & - \\
\hline & Wegener's granulomatosis & 1 & - & - & - & 1 & - \\
\hline & Underlying disease not reported & 4 & - & - & 1 & 3 & - \\
\hline & Total (rituximab) & $39(100 \%)$ & $4(10.3 \%)$ & $1(2.6 \%)$ & $10^{*}(25.6 \%)$ & $24(61.5 \%)$ & $-(0 \%)$ \\
\hline Alemtuzumab & B-CLL & 4 & - & - & $1^{*}$ & 3 & - \\
\hline Efalizumab & Psoriasis & 3 & 1 & - & - & 2 & - \\
\hline Ofatumumab & B-CLL & 1 & 1 & - & - & - & - \\
\hline Total & & $119(100 \%)$ & $31(26.1 \%)$ & $2(1.7 \%)$ & 12 (10.1\%) & $45(37.8 \%)$ & $29(24.4 \%)$ \\
\hline
\end{tabular}


reports from clinical trials. The number of reported suspected PML cases by medicinal product and underlying disease and the assessment according to the proposed case definition are presented in table 1. The majority of reports were notified for natalizumab $(n=72)$, followed by rituximab $(n=39)$. For efalizumab (Raptiva), for which the marketing authorisation in the European Union and in the USA was withdrawn in 2009 due to an unfavourable risk to benefit ratio, three suspected cases were reported. Four suspected cases were received for alemtuzumab and one case for ofatumumab.

In all, 45 reports (37.8\%) fulfilled the proposed case definition (levels 1-3) with sufficient diagnostic certainty, and 45 reports $(37.8 \%)$ were assessed as level 4, mainly because of missing information (42/45) with regard to the certainty of the PML diagnosis. In three reports, the given information was not compatible with levels $1-3$ and was not sufficient to exclude a PML. Missing or incomplete information was related to JCV DNA PCR in the CSF ( $\mathrm{n}=40), \operatorname{MRI}(\mathrm{n}=34)$ and (specific) clinical symptoms $(n=15)$. In four reports in which a PML was (clinically) ruled out according to the reporting physician, information about an alternative diagnosis was not available, and in four reports, information regarding brain biopsy/brain tissue sample was incomplete. Altogether, examination of brain tissue was performed in 11/119 (9.2\%) reports.

According to the case definition in 29 reports $(24.4 \%)$, all following therapy with natalizumab, PML was excluded, and an alternative diagnosis was established $(n=26$ : relapse, exacerbation, rebound or progression of $M S, n=1$ : each posterior reversible encephalopathy syndrome, stress reaction and aggravated depressive syndrome in combination with circulatory disorder).

Patient characteristics of levels 1-4 cases are presented in table 2. Nineteen patients $(21.1 \%)$ died. The percentage of reported fatalities differed among patient groups. In PML patients with underlying malignancies, the death rate was higher compared with patients with MS. Thus, the status of the malignant disease may have contributed to the unfavourable outcome.

Overall, there is an equal distribution of males and female subjects with regard to levels 1-4 PML reports. Of note, an inverse distribution of sex was reported for levels 1-4 cases following natalizumab and rituximab. This observation is most likely consistent with the sex-related frequency of the diseases covered by the respective indications. The age range of patients with levels $1-4$ is wide (23-83 years of age). The same is true of the duration of the $M A B$ treatment before onset of symptoms.

A range of different first symptoms has been reported in levels 1-4 PML cases (figure 1) indicating that PML clinical symptoms are not pathognomonic.

\section{DISCUSSION}

We propose a case definition which combines several diagnostic findings as well as clinical symptoms. We applied the proposed case definition to 119 suspected cases of PML following treatment with MABs. A total of 45 case reports were assessed as meeting the criteria for diagnostic certainty (levels 1-3); in 29 reports, a PML was excluded according to the case definition (level 5), and in 45 reports, no definite case ascertainment could be performed mainly due to missing information (level 4). It is notable that the amount of information concerning diagnostic certainty varied among the different MABs with generally more complete information available for natalizumab. This may be partly related to the established PML algorithm used for patients treated with natalizumab. The high percentage of level 4 reports

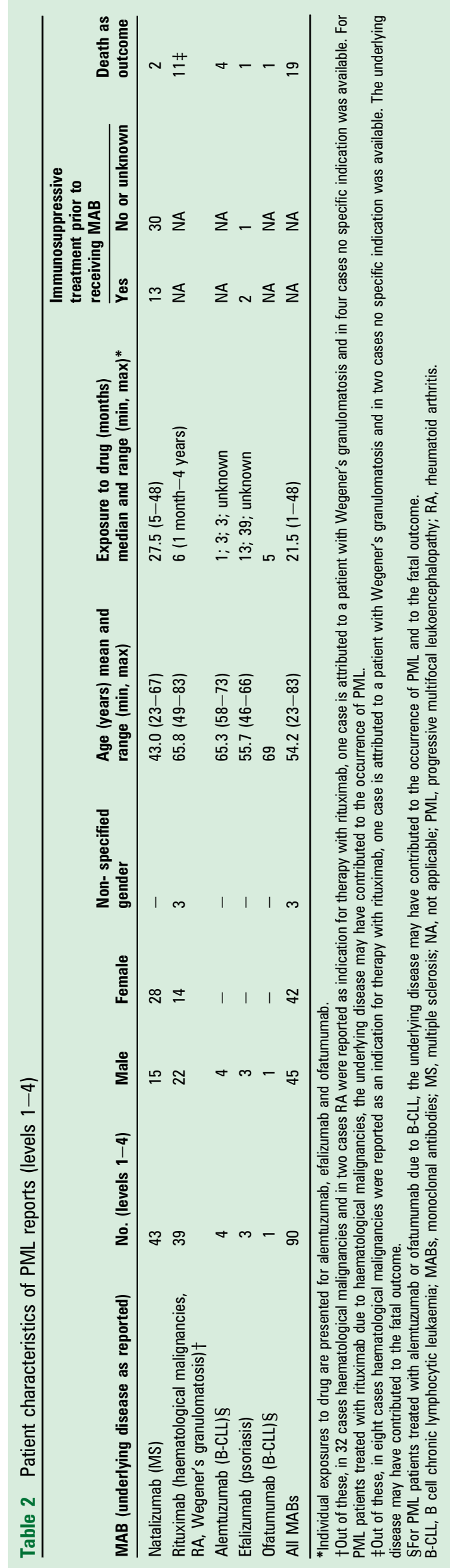


Figure 1 First symptoms of progressive multifocal leukoencephalopathy (PML) in levels 1-4 reports (referring to all mentioned monoclonal antibodies, patients may have $\geq 1$ onset symptom of PML). MS, multiple sclerosis.

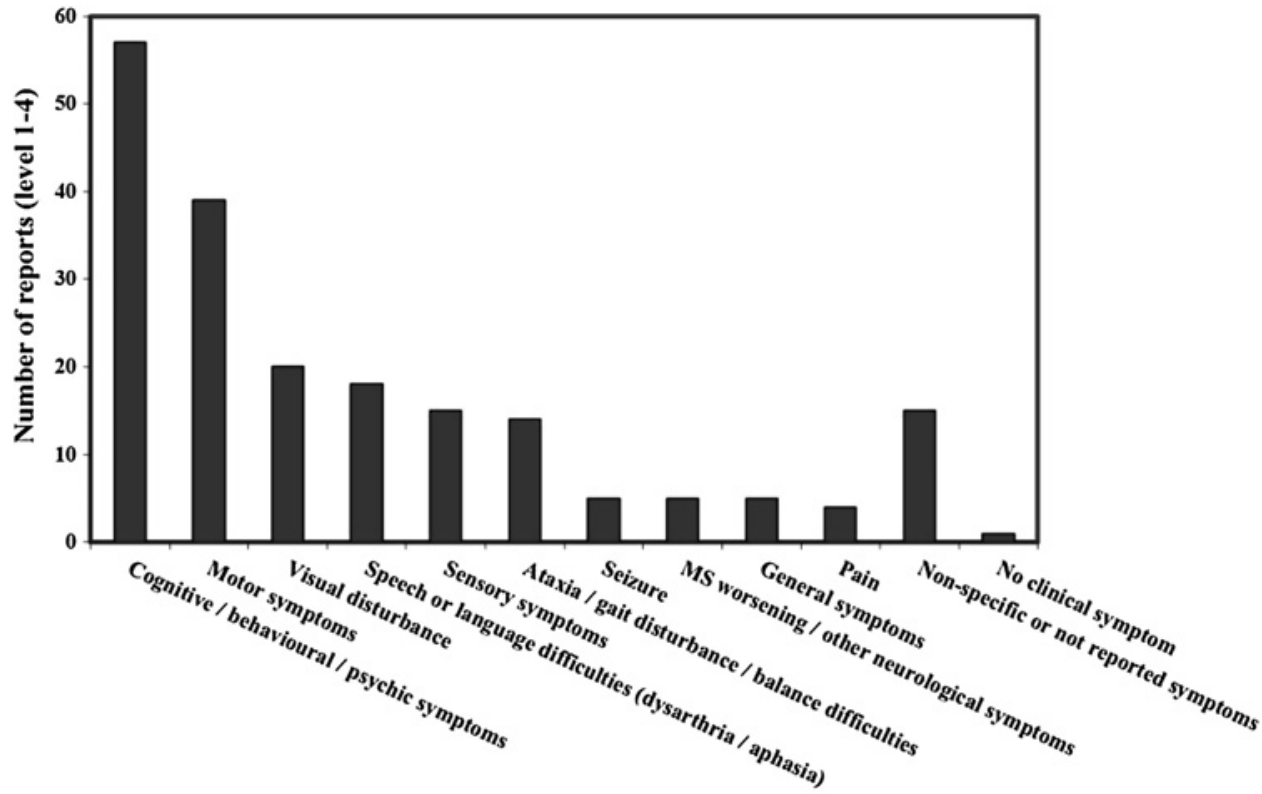

Reported first symptoms of PML, classified in categories

(37.8\%) underlines the importance of a PML case definition. The case definition provides guidance on essential data to be collected by physicians in clinical practice, and it facilitates the specific request for missing information by national competent authority and marketing authorisation holders.

Considering the complex diagnosis and the heterogeneous clinical presentation of PML, a case definition is a prerequisite for a thorough assessment of PML. The proposed case definition can be used for case ascertainment of suspected PML cases for various $\mathrm{MABs}$ covering a broad spectrum of indications. In addition, it will permit a common understanding of frequencies or reporting rates of PML associated with different MABs and thereby will facilitate comparisons among products. To our knowledge, this is the first published case definition for MABassociated PML. We applied the case definition retrospectively to reported suspected PML cases following treatment with MABs. A prospective testing of the diagnostic criteria will be a necessary next step. A further step will be an evaluation of the applicability of the case definition to non-MAB-associated PML.

We developed a dynamic case definition. The level of diagnostic certainty may change for a certain case subsequently to availability of more complete information (eg, multiple PCR or MRI results becoming available). Even in cases where the available information is not yet complete, the case definition provides a level of scientific certainty of the diagnosis. Thus, it is equally applicable to active as well as passive surveillance settings.

Several case definitions follow well-established, clinical definition formats as 'definite', 'probable' and 'possible' cases. The working group deliberately avoided using this terminology, because this grading can apply equally to causality assessment and diagnostic certainty. The proposed case definition focuses on diagnostic certainty, not on causality.

Detection of JCV in the CSF PCR is usually highly indicative of the diagnosis of PML. However, JCV PCR may be negative especially in early PML as well as during IRIS despite typical clinical and radiological features. Usually, clinical symptoms of PML trigger additional diagnostic examinations. It is acknowledged that there have been a few reports of PML diagnosed by routine MRI findings and subsequent CSF PCR positivity without evidence of clinical symptoms. In our analysis, PML was suspected only in one report in a patient without any clinical symptom(s) on the basis of a pathological MRI. However, as this constellation is considered to be the exception and only relevant if routine MRI is recommended for patients treated with a certain $\mathrm{MAB}$, we considered the triad of clinical symptoms consistent with PML, characteristic brain MRI and CSF PCR positivity as essential to meet level 1 criteria (if there is no evidence of PML from a brain biopsy or autopsy). PML cases without clinical symptoms but characteristic MRI and positive PCR for JCV DNA in the CSF will be classified as level 3 and are thus covered by the proposed case definition.

A two-step ELISA for detecting anti-JCV antibodies in serum and plasma was developed by Gorelik et al. ${ }^{2}$ The objective was to use the anti-JCV antibody serostatus as a tool for risk stratification of natalizumab-treated MS patients. At present, an evaluation of this approach for diagnostic purposes for PML is not available. Therefore, anti-JCV serostatus was not included in our case definition.

In conclusion, the proposed PML case definition permits data comparability among different medicinal products and different settings (clinical trials, postauthorisation safety studies and passive postmarketing surveillance). This case definition may also form a basis for meaningful risk analysis and communication of medicinal product safety for regulators and healthcare professionals.

Acknowledgements The authors thank Claudia Pönisch (Department of Safety of Medicinal Products and Medical Devices, Paul-Ehrlich-Institute, Langen) for queries in the adverse drug reaction database of the Paul-Ehrlich-Institute.

Contributors Contribution to the conception: all authors (working group discussion). Analysis and interpretation of the data: DM, JP, BKS. Drafting the manuscript: DM, JP, BKS. Critical revision of the manuscript for important intellectual content: $O A, R G$, $\mathrm{HPH}, \mathrm{HH}, \mathrm{BCK}$, WDL. Approval of the final version of the manuscript: all authors. Guarantorship and responsibility for the overall content of the manuscript: BKS. DM and JP contributed equally to the work.

Competing interests All authors have completed the Unified Competing Interest form at http://www.icmje.org/coi disclosure.pdf lavailable on request from the corresponding author) and declare RG and HPH have received financial support for the 
submitted work. RG has received consulting fees or honorariums from Biogen Idec, TEVA, Merck Serono, Novartis and Bayer Schering; the institution of RG has received grants from the above mentioned companies; RG has obtained financial support for travels to meetings for the study or other purposes from Biogen Idec and fees for participation in review activities from TEVA. HPH has received consulting fees or honorariums from Grifols and Baxter. In addition, relevant financial activities exist outside the submitted work: RG has received payment from Biogen Idec, TEVA, Merck Serono, Novartis and Bayer Schering for board membership, consultancy and lectures; the institution of RG has received grants from TEVA; RG has been paid from Novartis for the development of educational presentations; HPH has been paid from Biogen Idec, Novartis Pharma, Genzyme, Bayer HealthCare and Merck Serono for consultancy. There are no other relationships or activities that could appear to have influenced the submitted work.

Ethics approval The data referring to human subjects (suspected cases of progressive multifocal leukoencephalopathy following treatment with monoclonal antibodies) were reported to the Paul-Ehrlich-Institute as the national competent authority that is responsible for the safety of monoclonal antibodies in Germany according to German and European legislation. The data, which are included in the adverse drug reaction database of the Paul-Ehrlich-Institute, were analysed in this study. The database has been audited by the National Data Protection Officer. An Ethics Committee approval is not necessary for analysis of adverse drug reaction reports.

Provenance and peer review Not commissioned; externally peer reviewed.

\section{REFERENCES}

1. Egli A, Infanti L, Dumoulin A, et al. Prevalence of polyomavirus BK and JC infection and replication in 400 healthy blood donors. J Infect Dis 2009:199:837-46.

2. Gorelik L, Lerner M, Bixler S, et al. Anti-JC virus antibodies: implications for PML risk stratification. Ann Neurol 2010;68:295-303.

3. Delbue S, Branchetti E, Bertolacci S, et al. JC virus VP1 loop-specific polymorphisms are associated with favorable prognosis for progressive multifocal leukoencephalopathy. J Neurovirol 2009:15:51-6.

4. Berger JR, Houff SA, Major EO. Monoclonal antibodies and progressive multifocal leukoencephalopathy. MAbs 2009;1:583-9

5. Carson KR, Evens AM, Richey EA et al. Progressive multifocal leukoencephalopathy after rituximab therapy in HIV-negative patients: a report of 57 cases from the Research on Adverse Drug Events and Reports project. Blood 2009;113:4834-40.

6. Jalan P, Mahajan A, Pandav V, et al. Brentuximab associated progressive multifocal leukoencephalopathy. Clin Neurol Neurosurg. Published Online First: 1 April 2012. doi:10.1016/j.clineuro.2012.03.019

7. Brew BJ, Davies NW, Cinque P, et al. Progressive multifocal leukoencephalopathy and other forms of JC virus disease. Nat Rev Neurol 2010;6:667-79.

8. Yokoyama H, Watanabe T, Maruyama D, et al. Progressive multifocal leukoencephalopathy in a patient with B-cell lymphoma during rituximab-containing chemotherapy: case report and review of the literature. Int $\mathrm{J}$ Hematol 2008;88:443-7.
9. Vinhas de Souza M, Keller-Stanislawski B, Blake K, et al. Drug-induced PML: a global agenda for a global challenge. Clin Pharmacol Ther 2012;91:747-50.

10. Kohl KS, Bonhoeffer J, Braun MM, et al. The Brighton Collaboration: creating a global standard for case definitions (and guidelines) for adverse events following immunization. In: Henriksen K, Battles JB, Marks ES, et al, eds. Advances in patient safety: from Research to Implementation (Volume 2: Concepts and

Methodology). Rockville, MD: Agency for healthcare research and quality (US) 2005:87-102.

11. International Conference on Harmonisation of Technical Requirements for Registration of Pharmaceuticals for Human Use (ICH). Maintenance of the ICH guideline on clinical safety data management: data elements for transmission of individual case safety reports E2B(R2). 2001:1-25. http://www.ich.org/fileadmin/Public Web Site/ ICH_Products/Guidelines/Efficacy/E2B/Step4/E2B_R2_Guideline.p̄df laccessed 24 Apr 2012).

12. Tan CS, Koralnik IJ. Progressive multifocal leukoencephalopathy and other disorders caused by JC virus: clinical features and pathogenesis. Lancet Neurol 2010;9:425-37.

13. Clifford DB, DeLuca A, Simpson DM, et al. Natalizumab- associated progressive multifocal leukoencephalopathy in patients with multiple sclerosis: lessons from 28 cases. Lancet Neurol 2010:9:438-46.

14. Clifford DB, Ances B, Costello C, et al. Rituximab-associated progressive multifocal leukoencephalopathy in rheumatoid arthritis. Arch Neurol 2011:68:1156-64.

15. Kothary N, Diak IL, Brinker A, et al. Progressive multifocal leukoencephalopathy associated with efalizumab use in psoriasis patients. J Am Acad Dermatol 2011:65:546-51.

16. Schwab N, Ulzheimer JC, Fox RJ, et al. Fatal PML associated with efalizumab therapy: insights into integrin $\alpha \mathrm{L} \beta 2$ in JC virus control. Neurology 2012;78:458-67.

17. Biogen Idec. [Physician Information]. 2010:16-17. http://tysabri.de/index.php? inhalt=tysabri.sicherheitsmanagement (accessed 7 Feb 2012)

18. Shah R, Bag AK, Chapman PR, et al. Imaging manifestations of progressive multifocal leukoencephalopathy. Clin Radiol 2010;65:431-9.

19. Cinque P, Koralnik IJ, Gerevini S, et al. Progressive multifocal leukoencephalopathy in HIV-1 infection. Lancet Infect Dis 2009:9:625-36.

20. Metz I, Radue EW, Oterino A, et al. Pathology of immune reconstitution inflammatory syndrome in multiple sclerosis with natalizumab-associated progressive multifocal leukoencephalopathy. Acta Neuropathol 2012:123:235-45.

21. Tan K, Roda R, Ostrow L, et al. PML-IRIS in patients with HIV infection: clinical manifestations and treatment with steroids. Neurology 2009:72:1458-64.

22. Kuhle J, Gosert R, Bühler R, et al. Management and outcome of CSF-JC virus PCR-negative PML in a natalizumab-treated patient with MS. Neurology 2011;77:2010-16

23. Hellwig K, Gold R. Progressive multifocal leukoencephalopathy and natalizumab J Neurol 2011;258:1920-8.

24. Iacobaeus $\mathbf{E}$, Ryschkewitsch C, Gravell M, et al. Analysis of cerebrospinal fluid and cerebrospinal fluid cells from patients with multiple sclerosis for detection of JC virus DNA. Mult Scler 2009:15:28-35.

25. Tan CS, Ellis LC, Wüthrich $\mathrm{C}$, et al. JC virus latency in the brain and extraneura organs of patients with and without progressive multifocal leukoencephalopathy. $J$ Virol 2010:84:9200-9.

\section{Electrical injury to the brain}

A 37-year-old, previously healthy man was sent to our neurovascular outpatient clinic for a second opinion because of a presumed stroke at young age for which no obvious cause was found. The patient had global numbness and clumsiness of his left arm and numbness of the left side of his face, which had started suddenly 3 months prior to the visit, and had improved slightly. The complaints had started after he had touched live electricity cables with his left hand at a construction site. Initially, he also perceived white dots, with both eyes, on either side of his visual field during several days. A reduced sense of touch of the entire left arm and left side of his face, clumsiness of the left arm, and an elevated tendon reflex of the left knee were found on neurological exam. EMG revealed neuropathy of the left ulnar and median nerves. MRI scans four months after onset demonstrated a lesion with spotty appearance of the white matter in the right parietal area. MR angiography, ECG and extensive blood tests were normal.

\section{MULTIPLE CHOICES}

What is your diagnosis?

Ischaemic stroke from thromboembolism

Stroke caused by reversible vasoconstriction

Multiple sclerosis

Electrical brain injury

Migraine

\section{COMMENT}

The lesion has a spotty appearance of the MRI lesion, which is atypical for stroke, and is confined to white matter only, and outside the anatomical vascularisation areas (figure 1). Thus, stroke is highly unlikely, and the appearance of the lesion is more suggestive of the results from the electric shock. The initial visual complaints fit in with this hypothesis. The peripheral nerves, ipsilateral to the side of shock, and the contralateral upper white matter tracts are damaged, suggesting that this whole nerve tract has been injured. Nerves are susceptible to electrical injury, because they form the route of least resistance in the body. 